CDD: 025.527

\title{
QUAUDADE DE PRODUTOS E SERVICOS TURÍSTICOS EM EVENTOS: UMA PROPOSTA PARA ANÁLISE DE CONTEÚDOS DO TWITTER
}

\author{
QUALITY OF TOURISTIC PRODUCTS AND SERVICES IN EVENTS: \\ A PROPOSAL FOR CONTENT ANALYSIS ON TWITTER
}

\author{
Augusto José Waszczynskyj Antunes das Neve ${ }^{1}$ \\ Patricia Zeni Marchiori ${ }^{2}$
}

\begin{abstract}
Resumo: Ao se considerar a crescente adoção de mídias sociais e seu uso estratégico em organizações, o trabalho objetiva discutir o uso da análise de conteúdo no Twitter como recurso para a avaliação da qualidade percebida de produtos e serviços turísticos em eventos. Metodologicamente, o estudo se caracteriza como qualitativo, tendo caráter exploratório e descritivo. A partir do levantamento de literatura pertinente voltada à exploração dos impactos do Twitter, especialmente na atividade turística, sugeriu-se uma estrutura de análise de conteúdo - baseada na técnica Análise de Avaliação/Evaluation Assertion Analysis (EAA) - a qual foi aplicada a uma amostra de postagens realizadas por usuários do Twitter durante os Jogos Olímpicos de Londres de 2012. Tal estrutura, embora apresente aplicabilidade restrita por necessitar de mão de obra especializada e o investimento de horas de trabalho para a sua operacionalização, mostra-se útil para determinar as percepções/atitudes de usuários expressadas no Twitter em relação aos produtos e serviços turísticos consumidos quando da participação em eventos. Uma possibilidade de continuidade desta investigação estaria no efetivo levantamento e comparação de algoritmos que viabilizam a análise de conteúdo em ferramentas automatizadas, verificando-se o alinhamento destas a uma metodologia "clássica". Os resultados da investigação podem, igualmente, oferecer subsídios para a criação de uma ferramenta que alie a estrutura da EAA à eficiência da operacionalização de processos decorrentes da automação.
\end{abstract}

Palavras-chave: Análise representacional. Produtos e serviços turísticos. Mídias sociais. Eventos.

\begin{abstract}
It discusses the increasing adoption of social media to sustain strategic issues in organizations, and the use of content analysis technique applied to Twitter content perceived as an attitudinal resource regarding the quality of touristic products and services. It is an exploratory and descriptive research with a qualitative approach. It was suggested a framework for content analysis - based on the Evaluation Assertion Analysis (EAA) technique - which was applied to a sample of Twitter posts collected during the 2012 London Olympic Games. The proposed framework, although has a limited applicability due to specialized personnel and working hours requirements, proves to be helpful on determining the attitudes of Twitter users in relation to touristic products and services consumed. One possibility on continuing this research would be in the effective survey and comparison of algorithms that enable content analysis in automated tools, in order to check their alignment with a
\end{abstract}

\footnotetext{
${ }^{1}$ Universidade Federal do Paraná. Graduado em Turismo pela UFPR; Mestre em Ciência, Gestão e Tecnologia da Informação pela UFPR. Brasil. E-mail: augustojw@gmail.com

${ }^{2}$ Universidade Federal do Paraná. Bacharel em Biblioteconomia pela UFPR, Mestre em Ciência da Informação pela UFRJ/IBICT, Doutora em Comunicação ECA/USP. Professor Titular do Departamento de Ciência e Gestão da Informação da UFPR. Brasil E-mail: pzeni@ufpr.br

Recebido em: 10/05/2013 - Aceito em: 08/08/2013.
} 
"classical methodology". The results may also provide support to the creation of an alternative tool joining the EAA with the efficiency of operational processes resulting from automation

Keywords: Representational analysis. Tourism products and services. Social media.Events.

\section{INTRODUÇÃO}

No ano de 2008, durante o processo eleitoral para a definição do Presidente da República dos Estados Unidos da América, foi possível constatar a importância que as mídias sociais adquiriram nas decisões do cotidiano. Durante este evento, o ainda Presidente Barack Obama (reeleito em 2012) se comunicava com os seus apoiadores através do Twitter ${ }^{3}$ e do Facebook ${ }^{4}$. Um dos objetivos do candidato e de sua equipe de marketing era o de incentivar os jovens a se manifestarem durante o processo eleitoral e, desta forma, contribuírem como cidadãos na decisão do futuro político do país.

Mais recentemente, no início de 2011, o Twitter surgiu como um dos principais instrumentos usados pelos populares para se comunicarem entre si e com o restante do mundo quando da chamada "revolução egípcia", e consequente renúncia do ditador Hosni Mubarak.

Do ponto de vista corporativo, mídias sociais como o Twitter, por exemplo, têm adquirido alguma relevância no que concerne às estratégias de relacionamento. As organizações utilizam estas ferramentas não só para divulgarem e promoverem seus produtos e serviços, como também para dialogarem diretamente com os consumidores, esclarecendo dúvidas, desburocratizando a resolução de problemas e ajudando a criar vínculos de proximidade e de confiança.

Estas mudanças têm provocado alterações em diversas atividades da economia, inclusive na atividade turística. Nesta, essas transformações são constatadas quando relatos surgem em tais mídias que veiculam a opinião de usuários/consumidores de produtos e serviços turísticos ${ }^{5}$. Em tal processo, os

\footnotetext{
${ }^{3}$ O Twitter, fundado em 2006, permite ao usuário publicar mensagens (tweets), para os seus seguidores (followers), cujo conteúdo utiliza o formato de texto em até, no máximo, 140 caracteres, podendo ser acompanhado de vídeo, endereços eletrônicos ou fotos (TWITTER, 2012).

${ }^{4} \mathrm{O}$ Facebook é uma rede social na qual as pessoas se relacionam virtualmente com seus amigos, colegas de trabalho, colegas de estudo e grupos de convívio (FACEBOOK, 2013).

${ }^{5}$ Um produto turístico pode ser definido como "[...] o conjunto de atrativos, equipamentos e serviços turísticos acrescidos de facilidades, localizados em um ou mais municípios, ofertado de forma organizada por um determinado preço" (BRASIL, 2007, p. 17).
} 
consumidores assumem o papel de personagens ativos no monitoramento das atividades de que participam ou pretendem participar, podendo se tornar formadores de opinião em suas comunidades virtuais.

Para Bahl (2004), ao se apresentarem como motivadores de viagens, os eventos geram um fluxo adicional de consumidores para o local onde tais eventos ocorrem. Em outras palavras, os eventos provocam o surgimento de uma demanda real que se desloca nesse ou para um determinado espaço geográfico com a finalidade de consumir produtos e serviços turísticos ali existentes, os quais serão avaliados (sob distintos graus de satisfação) conforme a qualidade percebida decorrente da vivência deste consumo (ZITTA, 2009). Cesca (2008) esclarece que a realização de um evento implica planejar, elaborar e implementar um projeto de um acontecimento que visa manter ou modificar a imagem e o conceito de uma organização perante o seu público alvo e o mercado no qual se insere. Assim, se a qualidade percebida pelo turista for distinta da qualidade esperada, podem surgir sentimentos de frustração e insatisfação em relação à vivência como um todo.

Churchill e Peter (2010) especificam que os consumidores avaliam a qualidade dos produtos e serviços que consomem de acordo com, pelo menos, cinco critérios, a saber: aspectos tangíveis, confiabilidade, prontidão/presteza, garantia e empatia. Ao serem avaliados pelo consumidor de forma individual, estes critérios podem revelar a sua satisfação em relação aos produtos e serviços turísticos que usufruem.

Quando se considera especificamente o setor de eventos, os estudos que envolvem o microblog ${ }^{6}$ Twitter na área de Turismo têm apontado para o seu uso como fonte de informação para públicos diferentes daquele representado pelos seus participantes (EBNER et al., 2010); como instrumento de marketing (SHIMADA et. al., 2011); como meio de comunicação que dissemina mensagens de qualidade e relevantes relacionadas a eventos (BECKER; NAAMAN; GRAVANO, 2011); e na identificação de características e comportamentos relacionados ao público que acompanha ou participa de um determinado evento (LOTAN, 2011).

\footnotetext{
${ }^{6} \mathrm{Um}$ microblog é uma vertente das mídias sociais que oferece formas de produzir e disseminar conteúdos que apresentam um ciclo de vida curto e repetitivo (VIEWEG et al., 2010). Heverin e Zach (2010) o conceituam como uma ferramenta com as características de um blog, mas que permite aos seus usuários enviar breves atualizações de conteúdo por meio da web ou de dispositivos móveis.
} 
Conteúdos veiculados em microblogs podem expressar atitudes ou evocar agregados de sentimentos; fato este que tende a influenciar o ponto de vista dos usuários que partilham a plataforma em relação a produtos, serviços ou mesmo opiniões gerais (JANSEN et al., 2009). Para o autor, esta característica, aliada ao uso de linguagem chamativa e de fácil entendimento, tem contribuído para que os usuários venham trocando as ferramentas tradicionais de comunicação na internet (blogs ou correio eletrônico) por serviços de microblog.

Considerando este cenário, os intervenientes da atividade turística, sejam eles integrantes da iniciativa pública ou privada, reconhecem a oportunidade de monitorar e analisar o conteúdo das informações/opiniões produzidas pelos consumidores e disseminadas nas mídias sociais. Tais mídias podem se tornar ferramentas úteis na melhoria da qualidade de produtos e serviços turísticos já disponíveis para consumo/uso e também na elaboração de novos produtos/serviços voltados para exigências específicas, as quais são disseminadas nestes espaços de comunicação.

A coleta contínua e persistente destes conteúdos (e consequente análise) tende a demandar soluções tecnológicas. Algumas ferramentas ${ }^{7}$ estão disponíveis gratuitamente (a partir de cadastro), sendo que outras oferecem a modalidade gratuita com um limite no número de postagens para análise (versões demo ${ }^{8}$ ); outras, ainda, exigem pagamento para seu uso. Tais ferramentas utilizam algoritmos e métodos estatísticos e probabilísticos para a análise os quais nem sempre são explicitados (especialmente nas ferramentas de acesso gratuito).

Sob outra ótica complementar, se reconhece uma metodologia consolidada (e tida como "clássica”) para a análise de conteúdo, a qual consiste de técnicas e procedimentos que podem se voltar à descrição e mensuração de “... atitudes do locutor quanto aos objetos de que ele fala" (BARDIN, 2011, p. 203). Assim ao se identificar, no contexto desta investigação, o "locutor" como o usuário/participante de uma mídia social (no caso o Twitter), e os produtos e serviços turísticos em eventos como "objetos", pretendeu-se discutir o uso da análise de conteúdo como instrumento

\footnotetext{
${ }^{7}$ Um levantamento preliminar realizado no dia 16 de dezembro de 2012, no sítio eletrônico do Projeto Sentiment140 (http://help.sentiment140.com/other-resources), indicou a existência de aproximadamente 130 ferramentas voltadas à análise de informações disponíveis no Twitter. Tais ferramentas foram listadas e analisadas conforme suas condições de uso (níveis/limites de gratuidade) e existência de explicação sobre os algoritmos (ou demais métodos) que procedem a análise automática. ${ }^{8}$ Versão de demonstração [...]. É possível usar o programa por um tempo ou com apenas algumas funções disponíveis (FERREIRA, 2007).
} 
na avaliação da qualidade percebida de produtos e serviços turísticos em eventos.

$\mathrm{Na}$ sequência do artigo são apresentados os procedimentos metodológicos, incluindo-se uma descrição da técnica de análise de conteúdo Evaluative Assertion Analysis (EAA/Análise Avaliativa ou Análise Representacional) (BARDIN, 2011); a definição do ambiente de estudo e o detalhamento de encaminhamentos relativos à coleta dos posts/tweets; e respectiva análise. Em seguida listam-se os resultados que apoiam a discussão acerca das condições e vantagens/desvantagens do uso de tal técnica para o monitoramento da percepção da qualidade no contexto de eventos. Comentários adicionais sobre a pesquisa, demais resultados relevantes e possibilidades de estudos futuros são apresentados na seção final.

\section{PROCEDIMENTOS METODOLÓGICOS}

Os Jogos Olímpicos de Londres de 2012 foram definidos como ambiente de estudo, pois este foi o primeiro evento internacional onde as mídias sociais tiveram um papel de destaque na divulgação e no compartilhamento de informações entre os participantes, tendo sido definidas regras para o uso de tais mídias por parte de atletas e de demais indivíduos portadores de credencial oficial do evento (INTERNATIONAL OLYMPIC COMMITTEE, 2012).

A metodologia de análise de conteúdo foi considerada para o estudo uma vez que objetiva, a partir de um conjunto de técnicas parciais, diferentes e complementares entre si, explicar e sistematizar o conteúdo de uma determinada mensagem, atribuindo-lhe um ou mais significados por meio de análises dedutivas respaldadas pela sua origem (emissor), pelas suas consequências (efeitos) e pelo contexto onde foi produzida (BARDIN, 2011).

Bardin (2011) esclarece que a explicitação, a sistematização e exteriorização do conteúdo presente em uma mensagem, ocorrem dentro da análise de conteúdo por meio da realização de três etapas, a saber: a) pré-análise, onde são organizadas e sistematizadas as ideias; definem-se quais os documentos que serão investigados; retomam-se e, se necessário, reformulam-se as hipóteses e os objetivos inicialmente estabelecidos para o material coletado; e elaboram-se os indicadores que irão auxiliar na etapa final de interpretação; b) exploração do material, que compreende a análise sistemática (codificação) dos dados brutos coletados para se determinar o núcleo de 
compreensão do texto; e, c) tratamento dos resultados obtidos, inferência e interpretação.

Na primeira etapa (pré-análise) as categorias adotadas para a identificação da opinião dos consumidores em relação a serviços turísticos foram: alimentação, hospedagem, transporte e segurança. Pelo fato de o idioma inglês ser um dos três mais falados no mundo (ESTADÃO, 2012) e ser o idioma oficial do Reino Unido, a busca da informação baseou-se neste léxico. Agregaram-se às categorias iniciais outros itens de análise coletados no sítio oficial do evento na Internet. Por exemplo, a categoria “alimentação" foi desmembrada nos seguintes itens: london 2012 menu; restaurant; dining; breakfast; dinner; bar; pub; brasserie; grill; cafe; lunch; fish \& chips; food; drink. A categoria hospedagem, conforme os dados disponíveis no sítio do evento, desmembrou-se em: accomodation; hotel; hostel; self-catering; homestay; camping; caravans; accessible hotels. Para as demais categorias (transporte e segurança) seguiu-se o mesmo procedimento.

Para a obtenção e armazenamento dos tweets e posterior agregação às categorias/itens de análise definidos, fez-se uso do aplicativo da Microsoft тм Analytics for Twitter ${ }^{\circledR}$. A escolha deste aplicativo se justifica pela gratuidade (até um limite pré-definido de tweets); pela interface agradável; pela possibilidade de exportar os dados em formato de planilha de cálculo Microsoft ${ }^{\mathrm{TM}}$ Excel ${ }^{\circledR}$; e pelo o acesso aos resultados no formato de gráficos (além do numérico) (MICROSOFT, 2012).

A coleta de conteúdos ocorreu diariamente - e após as 20h (horário de Brasília) - durante o período oficial da realização dos Jogos Olímpicos (de 27 de Julho a 12 de Agosto de 2012) de forma a corresponder ao horário oficial de encerramento das atividades esportivas diárias dos Jogos Olímpicos (24h no horário de Londres). Os resultados desta etapa revelaram que os usuários do Twitter criaram e/ou compartilharam mais de nove mil posts a respeito de itens de alimentação (4.576 tweets), transportes (3.486 tweets), segurança (1.274 tweets) e hospedagem (375 tweets).

Após a coleta e armazenamento procedeu-se a exclusão de tweets em formato replicado (o re-tweet), pois estes representam apenas o compartilhamento de conteúdo publicado por outro usuário, sem que se acrescente conteúdo novo em relação ao original (TWITTER, 2012). Para os tweets válidos definiu-se que os termos de 
pesquisa no aplicativo assumiriam a nomenclatura "\#(nome do evento)" (ex.: “\#london2012”). Além do uso desta expressão de busca adotou-se, adicionalmente, o uso do operador booleano "and" seguido do item que se pretendia observar dentro de cada uma das categorias definidas para a análise. Por exemplo, para recuperar informação sobre o metrô de Londres durante os Jogos Olímpicos, a expressão de busca foi elaborada como: \#london2012 and underground. $\mathrm{O}$ uso de booleanos para a recuperação de informações é recomendado pelo próprio aplicativo quando se pretende procurar por termos, usuários ou tópicos em específico. Quando se utiliza o booleano "and", a busca retorna os resultados que incluem os dois termos pesquisados.

Em seguida, e devido ao ainda considerável número de tweets validados (4.440), optou-se pela definição de $\operatorname{amostras}^{9}$ de cada uma das categorias estabelecidas para a aplicação da técnica de análise de conteúdo. Por se tratar de uma amostragem estratificada, definiu-se que as categorias/itens de alimentação, hospedagem, transporte e segurança seriam subgrupos da população considerada válida para análise.

Ao se adotar a fórmula ${ }^{10}$

$$
n=\frac{\sigma^{2} p \cdot q \cdot N}{e^{2}(N-1)+\sigma^{2} p \cdot q}
$$

Para o cálculo de amostras em populações finitas obtiveram-se os seguintes resultados: 362 tweets relacionados a alimentação, 38 de hospedagem, 301 de transporte e 177 de segurança. Considerando-se que cada categoria agrega mais de um item de análise, decidiu-se trabalhar com a amostra dos itens mais representativos (com mais tweets válidos) de cada uma das categorias. Esta escolha, além de privilegiar assunto mais comentado/compartilhado dentro de cada uma das categorias, permitiu analisar cada um desses itens de forma específica, identificando de forma

\footnotetext{
${ }^{9}$ Em pesquisas sociais a estimativa de erro varia entre os $3 \%$ e os $5 \%$ enquanto o nível de confiança varia entre 68\% (um desvio padrão) e 99,7 (três desvios padrão) (GIL, 1999). Como se trata de uma população de 4.440 elementos $(\mathrm{N}=4.440)$, finita e inferior a 100.000 elementos, adotou-se um nível de confiança de $95,5 \%$ (dois desvios padrão; $\sigma=2$ ) e um erro máximo permitido de $5 \%(\mathrm{e}=5)$ para $\mathrm{o}$ cálculo das amostras de cada uma das categorias.

${ }^{10}$ Onde: $n$ é o tamanho da amostra; $\sigma^{2}$ o nível de confiança escolhido, expresso em números de desviopadrão; $p$ a porcentagem com a qual o fenômeno se verifica; $q$ a porcentagem complementar; $N$ o tamanho da população; $e$ o erro máximo permitido.
} 
mais pormenorizada as atitudes/percepções demonstradas pelos usuários em relação a estes. Os itens escolhidos foram food para a categoria de alimentação, hotel para hospedagem, bus para transporte e security para segurança.

Das cinco técnicas de análise de conteúdo ${ }^{11}$ optou-se pela Análise de Avaliação (ou Análise Representacional) para a segunda etapa do estudo, por se assumir que a problemática da investigação buscava “... medir as atitudes do locutor quanto aos objetos de que ele fala" (BARDIN, 2011, p. 203). Conhecida como Evaluative Assertion Analysis (EAA), esta técnica foi criada e desenvolvida por Osgood em 1959 e se fundamenta em conhecimentos da psicologia social que versam sobre a noção de atitude. Para Allport (1935, apud CAVAZZA, 2008, p.16), a atitude corresponde a "um estado mental ou neurológico de prontidão (readiness), organizado por meio da experiência, que exerce uma influência diretiva ou dinâmica sobre a resposta do indivíduo nos confrontos de todo objeto e toda situação com os quais entra em relação". Identificar o fundamento das atitudes, independentemente da forma que assumem quando verbalizadas, é o objetivo da EAA enquanto técnica da análise de conteúdo.

Entretanto, Bardin (2011) esclarece que, ao se realizar a EAA, nem todo o material pronto para ser analisado é considerado, pois é necessário escolher apenas os itens que exprimem uma avaliação em relação a um determinado objeto. Igualmente se deve especificar uma nomenclatura para a avaliação dos componentes relativos aos enunciados que serão sujeitos à EAA (BARDIN, 2011), a saber: a) os objetos de atitude (AO, do inglês Attitude Objects), que representam os itens sobre os quais recai a avaliação (pessoas, grupos, ideias, coisas, acontecimentos) e assumem a forma de substantivos ou pronomes pessoais; b) os termos avaliativos com significação comum (cm, do inglês evaluative common-meaning terms), responsáveis por qualificar os objetos de atitude e que assumem a forma de adjetivos, substantivos, advérbios formados a partir de adjetivos (ex.: lealmente), e alguns verbos (ex.: mentir, respeitar); e c) os conectores verbais (também denominados de "c"), que têm a função de fazer a ligação entre os objetos de atitude e os termos de qualificação.

\footnotetext{
11 Análise temática ou categorial; análise de avaliação ou representacional; análise da expressão; análise das relações; e análise da enunciação (BARDIN, 2011).
} 
$\mathrm{Na}$ terceira etapa da metodologia (tratamento dos resultados obtidos, inferência e interpretação) foram respeitadas as três fases da aplicação da EAA (BARDIN, 2011): a) a identificação e extração dos objetos de atitude (com o apoio do dicionário online de língua inglesa da Universidade de Princeton (http://wordnetweb.princeton.edu/perl/webwn); b) a normalização dos enunciados (obtenção de afirmativas e padronização do conteúdo dos tweets em frases sob a forma sintática mais elementar (ator-ação-complemento); e c) a codificação, ou seja, a atribuição de um direcionamento positivo, negativo ou neutro, e de um valor para a significação comum “cm" e para os conectores verbais "c", buscando facilitar a identificação das atitudes exteriorizadas pelos usuários nos posts criados e disponibilizados no Twitter.

\section{DESCRIÇÃO E ANÁLISE DOS RESULTADOS}

Da amostra de 362 tweets relacionados a alimentação, 38 de hospedagem, 301 de transporte e 177 de segurança foram escolhidos os itens food para a categoria de alimentação, hotel para hospedagem, bus para transporte e security para segurança. A estes foram aplicadas as etapas da EAA conforme o descrito a seguir.

Primeiramente, procedeu-se à identificação, classificação e direcionamento dos objetos de atitude ( $\mathrm{AO})$, termos avaliativos com significação comum $(\mathrm{cm})$ e conectores verbais (c). $\mathrm{Na}$ impossibilidade de se demonstrar o processo para o conjunto total de tweets, optou-se por apresentar um exemplo deste passo e a discussão decorrente (QUADRO 1).

QUADRO 1: Exemplo de identificação e classificação de conteúdos em tweets

\begin{tabular}{|c|l|c|}
\hline \multirow{2}{*}{ TWEET } & $\begin{array}{c}\text { IDENTIFICAÇÃO E CLASSIFICAÇÃO } \\
\text { DE CONTEÚDOS }\end{array}$ & $\begin{array}{c}\text { DIRECIONAMENTO } \\
\text { DOS CONTEÚDOS }\end{array}$ \\
\hline \multirow{2}{*}{$\begin{array}{c}\text { Getting food and } \\
\text { drink in order for } \\
\text { \#London2012 } \\
\text { opening ceremony }\end{array}$} & $\begin{array}{l}\text { Getting - conector verbal (c) } \\
\text { food and drink in order for -termos } \\
\text { avaliativos de significação comum (cm) }\end{array}$ & $\begin{array}{l}\text { \#London 2012 opening ceremony - objeto } \\
\text { de attitude (AO) }\end{array}$ \\
\hline
\end{tabular}

FONTE: os autores.

É possível verificar, ao se analisar sintaticamente o tweet, que o usuário que o criou/compartilhou está preparando comida e bebida para poder acompanhar a cerimônia de abertura das Olimpíadas de Londres 2012. Desta forma, \#London 2012 
opening ceremony (cerimônia de abertura de Londres 2012) surge como o objeto de atitude, pois é por causa dele que o usuário toma uma ação. Por se tratar de um acontecimento que nem todos os usuários assumiriam um sentimento positivo, optouse por atribuir um direcionamento neutro. $\mathrm{O}$ conector verbal getting indica que o usuário está preparando/conseguindo algo. Como o verbo to get (em português, obter) depende do contexto onde se insere para ter um direcionamento positivo ou negativo, também se optou por lhe atribuir neutralidade. Por sua vez, o conector verbal (food and drink in order for) indica que a comida e a bebida estão ficando prontas/em ordem para serem consumidas. Como é antecedido pelo verbo to get, o conector verbal adquire um direcionamento positivo.

Entretanto, nem sempre é possível distinguir todos estes conteúdos dentro de um tweet (QUADRO 2) .

QUADRO 2: Exemplo de identificação e classificação parcial de conteúdos em tweets

\begin{tabular}{|c|l|c|}
\hline \multirow{2}{*}{ TWEET } & $\begin{array}{c}\text { IDENTIFICAÇÃO E CLASSIFICAÇÃO } \\
\text { DE CONTEÚDOS }\end{array}$ & $\begin{array}{c}\text { DIRECIONAMENTO } \\
\text { DOS CONTEÚDOS }\end{array}$ \\
\hline \multirow{2}{*}{$\begin{array}{c}\text { Wine, Mexican food } \\
\text { and \#London } 2012\end{array}$} & $\begin{array}{l}\text { Wine, Mexican food }- \text { termos avaliativos de } \\
\text { significação comum }(\mathrm{cm})\end{array}$ & Neutro \\
\cline { 2 - 3 } & \#London $2012-$ objeto de attitude (AO) & Neutro \\
\hline
\end{tabular}

FONTE: os autores.

De acordo com este exemplo, não se verifica a existência de um conector verbal de forma explícita. Ainda assim, com base no significado de cada uma das palavras é possível verificar que o usuário está preparando comida mexicana acompanhada de vinho para ver as Olimpíadas. Se vinho, comida mexicana e as Olimpíadas apresentam um significado positivo ou negativo, depende do conector verbal e do contexto no qual eles são servidos, degustados e observados. Por este motivo optou-se por atribuir neutralidade tanto para o objeto de atitude quanto para o conector verbal.

Seguiu-se a normalização dos enunciados de forma a obter afirmativas que descrevam a intenção dos tweets e para que o conteúdo destes se tornasse passível de análise. O Quadro 3 apresenta a normalização dos tweets anteriormente exemplificados. 
QUADRO 3: Exemplo de normalização de enunciados relacionados a conteúdos de tweets

\begin{tabular}{|c|c|c|c|}
\hline TWEET & $\begin{array}{c}\text { IDENTIFICAÇÃO E } \\
\text { CLASSIFICAÇÃO DE } \\
\text { CONTEÚDOS }\end{array}$ & $\begin{array}{l}\text { DIRECIONAMENTO } \\
\text { DOS CONTEÚDOS }\end{array}$ & $\begin{array}{c}\text { NORMALIZAÇÃO DO } \\
\text { ENUNCIADO }\end{array}$ \\
\hline \multirow{3}{*}{$\begin{array}{l}\text { Getting food and } \\
\text { drink in order for } \\
\text { \#London } 2012 \\
\text { opening } \\
\text { ceremony }\end{array}$} & $\begin{array}{l}\text { Getting - conector verbal } \\
\text { (c) }\end{array}$ & Neutro & \multirow{3}{*}{$\begin{array}{l}\text { O usuário deixa alimentos } \\
\text { e bebidas em ordem para a } \\
\text { cerimônia de abertura de } \\
\text { Londres } 2012\end{array}$} \\
\hline & $\begin{array}{l}\text { food and drink in order } \\
\text { for -termos avaliativos } \\
\text { de significação comum } \\
(\mathrm{cm})\end{array}$ & Positivo & \\
\hline & $\begin{array}{l}\text { \#London } 2012 \text { opening } \\
\text { ceremony }- \text { objeto de } \\
\text { attitude (AO) }\end{array}$ & Neutro & \\
\hline \multirow{2}{*}{$\begin{array}{l}\text { Wine, Mexican } \\
\text { food and } \\
\text { \#London } 2012\end{array}$} & $\begin{array}{l}\text { Wine, Mexican food - } \\
\text { termos avaliativos de } \\
\text { significação comum }(\mathrm{cm})\end{array}$ & Neutro & \multirow{2}{*}{$\begin{array}{l}\text { O usuário escreve vinho, } \\
\text { comida mexicana e } \\
\text { Londres } 2012\end{array}$} \\
\hline & $\begin{array}{l}\text { \#London } 2012 \text { - objeto } \\
\text { de attitude (AO) }\end{array}$ & Neutro & \\
\hline
\end{tabular}

FONTE: os autores.

Conforme descrito nos procedimentos metodológicos, a normalização dos enunciados permite transformar os tweets em frases sob uma sintaxe mais elementar (ator-ação-complemento). A adoção deste formato, além de promover a atribuição de um direcionamento para o tweet como um todo, permite suprimir a falta de um conector verbal em alguns deles conforme anteriormente explicitado, facilitando atribuição de um significado neutro, positivo ou negativo.

Após a normalização dos enunciados, atribuiu-se a cada tweet um direcionamento (positivo, negativo ou neutro) (QUADRO 4).

QUADRO 4: Exemplo de direcionamento de enunciados relacionados a conteúdos de tweets

\begin{tabular}{|c|c|c|c|c|}
\hline TWEET & $\begin{array}{c}\text { IDENTIFICAÇÃO E } \\
\text { CLASSIFICAÇÃO } \\
\text { DE CONTEÚDOS }\end{array}$ & $\begin{array}{l}\text { DIRECIONAMENTO } \\
\text { DOS CONTEÚDOS }\end{array}$ & $\begin{array}{l}\text { NORMALIZAÇÃO } \\
\text { DO ENUNCIADO }\end{array}$ & $\begin{array}{l}\text { DIRECIONAMENTO } \\
\text { DO ENUNCIADO }\end{array}$ \\
\hline \multirow{3}{*}{$\begin{array}{c}\text { Getting } \\
\text { food and } \\
\text { drink in } \\
\text { order for } \\
\text { \#London20 } \\
12 \text { opening } \\
\text { ceremony }\end{array}$} & $\begin{array}{l}\text { Getting }- \text { conector } \\
\text { verbal (c) }\end{array}$ & Neutro & \multirow{3}{*}{$\begin{array}{l}\text { O usuário deixa } \\
\text { alimentos e bebidas } \\
\text { em ordem para a } \\
\text { cerimônia de abertura } \\
\text { de Londres } 2012\end{array}$} & \multirow{3}{*}{ Positivo } \\
\hline & $\begin{array}{lr}\text { food and } & \text { drink in } \\
\text { order for } & \text {-termos } \\
\text { avaliativos } & \text { de } \\
\text { significação } & \text { comum } \\
(\mathrm{cm}) & \\
\end{array}$ & Positivo & & \\
\hline & $\begin{array}{lr}\text { \#London } & 2012 \\
\text { opening ceremony - } \\
\text { objeto de attitude } \\
(\mathrm{AO})\end{array}$ & Neutro & & \\
\hline \multirow{2}{*}{$\begin{array}{c}\text { Wine, } \\
\text { Mexican } \\
\text { food and } \\
\text { \#London20 } \\
12\end{array}$} & $\begin{array}{l}\text { Wine, Mexican food- } \\
\text { termos avaliativos de } \\
\text { significação comum } \\
(\mathrm{cm})\end{array}$ & Neutro & \multirow{2}{*}{$\begin{array}{l}\text { O usuário escreve } \\
\text { vinho, comida } \\
\text { mexicana e Londres } \\
2012\end{array}$} & \multirow[t]{2}{*}{ Neutro } \\
\hline & $\begin{array}{l}\text { \#London } 2012- \\
\text { objeto de attitude } \\
\text { (AO) }\end{array}$ & Neutro & & \\
\hline
\end{tabular}

FONTE: os autores. 
É possível verificar que a atribuição do direcionamento aos enunciados elaborados com base nos tweets é influenciada pelo direcionamento atribuído ao conector verbal (c), ao termo avaliativo de significação comum $(\mathrm{cm})$ e ao objeto de atitude (AO), identificados e classificados por meio técnica EAA. Mais especificamente, são os termos avaliativos de significação comum que apresentam um papel relevante nesta etapa. Caso o tweet apresente um "cm" que é complementado pela ação do verbo, a sua positividade, negatividade ou neutralidade é identificável. Caso não haja a presença de um conector verbal que indique a ação do usuário em relação à atitude explicitada no tweet enfrenta-se uma dificuldade na atribuição de um direcionamento, o que acarreta um relato inexato do conteúdo exteriorizado pelo usuário.

Identificou-se, adicionalmente, que o direcionamento do enunciado permite determinar a atitude demonstrada pelo usuário no Twitter em relação às categorias e itens propostos para análise. Desta forma - considerando as amostras selecionadas para análise e as condições apresentadas nos exemplos acima - foram mapeadas as atitudes explicitadas para as quatro categorias e itens selecionados (QUADRO 5)

QUADRO 5: Atitudes de usuários representadas por meio de tweets

\begin{tabular}{|c|c|c|c|c|}
\hline \multirow{2}{*}{ CATEGORIA } & \multirow{2}{*}{ ITEM } & \multicolumn{2}{|c|}{ ATITUDES REPRESENTADAS PELOS TWEETS } \\
\cline { 3 - 5 } & ANALISADO & Positiva & Negativa & Neutra \\
\hline Alimentação & food & 178 & 131 & 53 \\
\hline Hospedagem & hotel & 21 & 5 & 12 \\
\hline Transporte & bus & 124 & 81 & 96 \\
\hline Segurança & security & 113 & 40 & 24 \\
\hline
\end{tabular}

FONTE: os autores.

De acordo com os resultados obtidos por esta técnica de análise de conteúdo, os itens food, hotel, bus e security foram percebidos de forma positiva (em comparação com as representações de atitudes negativas ou neutras). Neste contexto, é possível afirmar que a comida, os hotéis, os ônibus e a segurança - enquanto produtos e serviços turísticos disponibilizados para consumo durante os Jogos Olímpicos de Londres 2012 - foram considerados como satisfatórios de acordo com as atitudes dos usuários, assim como exteriorizadas na mídia social Twitter. Contudo, apenas o item security pode ser considerado como satisfatório, pois apresenta mais de metade das atitudes avaliadas como positivas (64\%) contra $23 \%$ de atitudes negativas e $13 \%$ de neutras. Os demais itens foram avaliados satisfatoriamente, mas todos com 
porcentagens de atitudes positivas inferiores a 50\% (food, 49\%; hotel, 32\%; bus, $41 \%$ ). Destaca-se também o fato do item bus ter porcentagem superior de atitudes neutras em relação às atitudes negativas, o que pode indicar que os usuários tenham exteriorizado mais opiniões informativas do que negativas em relação aos ônibus disponibilizados durante os Jogos Olímpicos de Londres 2012.

\section{CONSIDERAÇÕES FINAIS}

Ao permitirem uma maior interatividade entre os seus usuários, as mídias sociais (e o Twitter, como exemplificado neste estudo) têm permitido manifestações de amplo alcance em relação a atividades, experiências e consumo de produtos e serviços. A opinião de um usuário pode influenciar as escolhas de compra de outros participantes da rede/mídia. Neste espaço, um elogio pode provocar uma maior procura por um determinado produto ou serviço, enquanto uma crítica pode afastar o consumidor do consumo de um determinado bem.

No segmento de eventos turísticos, o mapeamento da percepção da qualidade de serviços e produtos por parte dos consumidores pode auxiliar as organizações na avaliação da qualidade da oferta, projetando ações ou realizando alterações visando adequar a oferta às necessidades da demanda. $\mathrm{O}$ aproveitamento de conteúdos disseminados por participantes de eventos que são usuários do Twitter se apresenta como uma oportunidade estratégica.

No que respeita à aplicação da técnica EAA (entre as disponíveis na metodologia de análise de conteúdo) para este mapeamento, as seguintes considerações merecem atenção. A primeira delas se refere ao fato de que a aplicação da técnica depende majoritariamente do pesquisador. Esta característica confere alguma liberdade na execução da análise e alguma capacidade adaptativa aos objetivos propostos com a sua aplicação. Entretanto, devido às diversas etapas - a definição de "c", "cm" e "AO"; a necessidade de se recorrer a um dicionário; a atribuição de direcionamentos de acordo com o contexto onde estão inseridos; e a elaboração de afirmativas que definam a atitude do usuário em relação ao que este opina - a aplicação desta técnica se torna um processo criterioso e demorado. Uma segunda consideração se refere ao tempo despendido na análise. Durante o processo, cada tweet tomou aproximadamente $2 \mathrm{~min} 24 \mathrm{seg}$ de análise, o que descarta o uso da 
técnica para situações com prazos exíguos. Neste particular, um grupo de pesquisadores/analistas poderia ser contratado, o que acarretaria custos adicionais e levaria a novas ponderações sobre a relação custo/benefício do uso da técnica. Ainda que a morosidade (e/ou o custo) de uso da técnica possa ser considerada como um fator negativo, esta se apoia em etapas interdependentes e indissociáveis que lhe conferem a coerência e a consistência derivada de um método científico consolidado.

Uma possibilidade de continuidade desta investigação estaria no efetivo levantamento e comparação de algoritmos em ferramentas automatizadas voltadas para a análise de tweets e o alinhamento destas a uma metodologia clássica. $\mathrm{O}$ presente estudo pode, igualmente, oferecer subsídios para o desenvolvimento de uma ferramenta que apresente comportamento analítico semelhante ao proposto pela EAA aliando os procedimentos preconizados pela técnica à eficiência de operacionalização de processos decorrente da automação. 


\section{REFERÊNCIAS}

BAHL, M. Turismo e eventos. Curitiba: Protexto, 2004.

BARDIN, L. Análise de conteúdo. São Paulo: Edições 70, 2011.

BECKER, H.; NAAMAN, M.; GRAVANO, L. Selecting quality Twitter content for events. In: INTERNATIONAL AAAI CONFERENCE ON WEBLOGS AND SOCIAL MEDIA, 5., 2011, Barcelona. Proceedings... Menlo Park: AAAI Press, 2011. Disponível em: <http://sm.rutgers.edu/pubs/becker82-icwsm2011.pdf〉. Acesso em 15 ago. 2012.

BRASIL, Ministério do Turismo. Programa de Regionalização do Turismo Roteiros do Brasil: Módulo Operacional 8 - Promoção e Apoio à Comercialização. Brasília, D.F. : Governo Federal, 2007.

CAVAZZA, N. Psicologia das atitudes e das opiniões. São Paulo: Edições Loyola, 2008.

CESCA, C. G. G. Organização de eventos: manual para planejamento e execução. 9. ed. São Paulo: Summus, 2008.

CHURCHILL, G. A. Jr.; PETER, J. P. Marketing: criando valor para o cliente. 2.ed. São Paulo: Saraiva, 2010.

EBNER, M. et al. Getting granular on twitter: tweets from a conference and their limited usefulness for non-participants. IFIP Advances in Information and

Communication Technology, v. 324, p. 102-113, 2010. Disponível em:

<http://www.springerlink.com/content/6n574q4p3098x68p/>. Acesso em 03 jul. 2012.

ESTADÃO. Estadão. Disponível em: <http://www.estadao.com.br>. Acesso em: 08 ago. 2012.

FACEBOOK. Facebook. Disponível em: <http://www.facebook.com>. Acesso em: 21 mar. 2013.

FERREIRA, L. Software livre, freeware, shareware, copyleft: entenda as licenças de software. Disponível em:

<http://tecnologia.uol.com.br/ultnot/2007/12/20/ult4213u266.jhtm>. Acesso em: 23 set. 2013.

GIL, A. C. Métodos e técnicas de pesquisa social. 5. ed. São Paulo: Atlas, 1999.

HEVERIN, T.; ZACH, L. Twitter for city police department information sharing. In: ASIS\&T NAVIGATION STREAMS IN AN INFORMATION ECOSYSTEM, 2010, Pittsburgh. Proceedings... Silver Springs: American Society for Information Science 
,2010. Disponível em:

<http://www.thomasheverin.com/uploads/4/6/5/8/4658640/twitter_city_police_depart ment_2010.pdf>. Acesso em: 28 mar. 2012.

INTERNATIONAL OLYMPIC COMMITTEE. IOC Social Media, Blogging and Internet Guidelines for participants and other accredited persons at the London 2012 Olympic Games. Disponível em:

<http://www.olympic.org/Documents/Games_London_2012/IOC_Social_Media_Blo gging_and_Internet_Guidelines-London.pdf>. Acesso em: 13 ago. 2012.

JANSEN, B. et al. Twitter power: tweets as electronic word of mouth. Journal of the American Society for Information Science and Technology, v. 60, n. 11, 2009.

Disponível em:

<http://www.cs.rochester.edu/twiki/pub/Main/HarpSeminar/Twitter_power-

_Tweets_as_electronic_word_of_mouth.pdf >. Acesso em: 31 jul. 2012.

LOTAN, G. Mapping information flows on twitter. In: INTERNATIONAL AAAI CONFERENCE ON WEBLOGS AND SOCIAL MEDIA, 5., 2011, Barcelona.

Proceedings... Menlo Park: AAAI Press, 2011. Disponível em:

<http://www.aaai.org/ocs/index.php/ICWSM/ICWSM11/paper/view/3838>. Acesso em: 01 jul. 2012.

MICROSOFT. Microsoft. Disponível em: <http://www.microsoft.com>. Acesso em: 04 jul. 2012.

SHIMADA, K. et al. Analyzing tourism information on twitter for a local city. In: ACIS INTERNATIONAL SYMPOSIUM ON SOFTWARE AND NETWORK ENGINEERING, 1., 2011, Seul. Proceedings... Seul: Seoul National University, 2011. Disponível em:

$<\mathrm{http}$ ://ieeexplore.ieee.org/stamp/stamp.jsp?tp=\&arnumber=6150078>. Acesso em: 03 abr. 2012

TWITTER. Twitter. Disponível em: <http://www.twitter.com>. Acesso em: 01 jun. 2012.

VIEWEG, S et al. Microblogging during two natural hazards events: what twitter may contribute to situational awareness. In: SIGCHI CONFERENCE ON HUMAN FACTORS IN COMPUTING SYSTEMS, 2010, Atlanta. Proceedings... New York: ACM, 2010. Disponível em:

<http://www.pensivepuffin.comwww.pensivepuffin.com/dwmcphd/syllabi/insc547_w i11/papers/microblog/vieweg.et.al.TwitterAwareness.CHI10.pdf>. Acesso em: 16 jun. 2012.

ZITTA, C. Organização de eventos: da ideia à realidade. 3.ed. Brasília: Editora Senac-DF, 2009. 
Como citar este artigo:

NEVE, Augusto José Waszczynskyj Antunes das; MARCHIORI, Patricia Zeni. Qualidade de produtos e serviços turísticos em eventos: uma proposta para análise de conteúdos do twitter. Rev. digit. bibliotecon. cienc. inf., Campinas, SP, v.12, n.1, p.61-77, jan/abr. 2014. ISSN 1678-765X. Disponível em: <http://www.sbu.unicamp.br/seer/ojs/index.php/rbci>. Acesso em: 30 jan. 2014. 